\title{
OPTIMIZED ENERGY ALLOCATION IN BATTERY POWERED IMAGE SENSOR NETWORKS
}

\author{
Chao Yu, Gaurav Sharma \\ Electrical and Computer Engineering Dept., \\ University of Rochester, Rochester NY 14627
}

\begin{abstract}
We investigate energy allocation strategies in image sensor networks for the purpose of maximizing the network operational lifetime. For the application scenarios that we consider, visual coverage over a monitored region is obtained by deploying wireless, battery-powered image sensors. Each sensor camera provides coverage over a part of the monitored region and a central processor coordinates the sensors in order to gather required visual data. We characterize the network lifetime as a stochastic random variable that depends upon the coverage geometry for the sensors and the distribution of data requests over the monitored region. Using this characterization we consider optimized strategies for energy allocation among the sensors that maximize the expected network lifetime. The formulation naturally leads to a max-min optimization problem that aims to maximize the duration of coverage for the most critical region for which the available energy is the least. We transform this problem into an equivalent linear programming problem, leading to a computationally efficient solution. The effectiveness of the proposed energy allocation strategy is validated by simulations.
\end{abstract}

Index Terms - image sensor network, network lifetime, energy allocation, linear programming

\section{INTRODUCTION}

Image/Visual sensor networks (VSNs) have recently emerged as an important subclass of wireless sensor networks $[1,2]$. The individual sensors in these networks consist of battery powered nodes that integrate a camera with wireless communication and networking capabilities. Applications of these VSNs include surveillance, human (patient) monitoring, and telepresence etc. Battery operations may be mandated in several applications, for instance, for monitoring an urban area following a natural disaster that causes power outage. Since the objective is to gather visual data from the sensors, the deployment of the sensor nodes is often constrained by the availability of mounting locations that are well-suited for capturing imagery (e.g. lamp posts, power line poles, corners of buildings etc). A consequence of these constraints is that the allocation of available energy among the sensors can have a significant impact on the network lifetime and the optimization of the energy allocation is therefore of interest.

The problem of optimal energy allocation is analogous to other wireless sensor network's where sensor locations are constrained [3, 4], with two important distinctions that justify our investigation.

This work is supported in part by the National Science Foundation under grant number ECS-0428157.
Firstly, unlike most other wireless sensor networks, meaningful definitions of lifetime for a VSN must take into account the visual coverage provided by the network $[5,6]$, which is determined by camera (and scene) geometry and not by sensor proximity alone. Secondly, in typical uses of VSNs, only a subset of the data is of interest, e.g. a surveillance network only interested in the moving objects. The impact of the stochastic nature of the data requests imposes extra challenges on performance analysis of the energy allocation problems. Our formulation addresses both of these aspects. We represent the network lifetime as a random variable (r.v.) that depends on the coverage geometry and on the distribution of user requests. This modeling of user requests leverages our recent work on sensor scheduling in a similar application scenario [7]. Based on the modeling of network lifetime, we formulate the lifetime-maximizing energy allocation strategy as an optimization problem and propose a linear programming (LP) solution.

Though our formulation is valid for several classes of VSNs, for concrete discussion, here we consider optimal energy allocation under an application scenario illustrated in Fig. 1, where image sensors are deployed to provide visual coverage over a monitored region. The network allows users to navigate around the monitored region by specifying a desired viewpoint (position and direction) that varies over time. The user's viewpoint determines the part of the scene that should be captured and transmitted to the user. The desired view at the viewpoint is synthesized at a central processor $(\mathrm{CP})$ by combining parts of the image sent from selected cameras. Given the total available energy, we investigate energy allocation strategies to distribute energy among these sensors with a view to maximizing the lifetime of the network.

\section{PROBLEM FORMULATION}

In this section, we present a formal description of our problem setting and formulate the energy allocation problem. Our setting is identical to that in [7], however, we address a different problem in this paper. The target plane $\mathcal{R}$ is monitored by $N$ Cameras $\left\{\mathcal{C}_{j}\right\}_{j=1}^{N}$. Each camera $\mathcal{C}_{j}$ is batteried with energy $w_{j}$ and covers a sub-region of $\mathcal{R}$ denoted by $\mathcal{V}_{j}$. We uniformly divide $\mathcal{R}$ into $M_{r}$ blocks $\left\{\mathcal{R}_{i}, i \in\left[M_{r}\right]\right\}$, where $[a]$ represent the set $\{1,2, \ldots, a\}$. We represent the coverage geometry of the cameras in terms of this discretized representation, and define a coverage matrix $\mathbf{B}^{r} \in \mathbb{R}^{M_{r} \times N}$ as

$$
\mathbf{B}_{i, j}^{r} \stackrel{\text { def }}{=} \mathbf{I}\left(\mathcal{R}_{i} \subseteq \mathcal{V}_{j}\right),
$$

where subscripts $i, j$ respectively denotes the row and column index of the matrix, and $a \subseteq b$ indicates region(set) $a$ lies within region(set) $b$, we also use $\mathbf{I}(\mathcal{A})$ to represent the indicator function: $\mathbf{I}(\mathcal{A})=1$ when $\mathcal{A}$ is true and 0 otherwise. The subset of cameras 


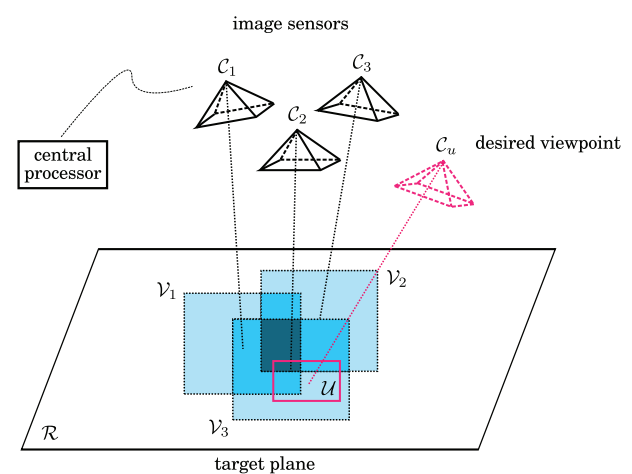

Fig. 1. The target plane $\mathcal{R}$ is monitored by cameras $\left\{\mathcal{C}_{j}\right\}_{j=1}^{N}$. The user requests a desired view $\mathcal{U}$, the $\mathrm{CP}$ selects a subset of cameras to provide data and synthesize the desired view. Regions that are covered by more cameras appear darker. Figure adopted from [7].

that cover block $\mathcal{R}_{i}$ is denoted by

$$
\lambda^{r}(i) \stackrel{\text { def }}{=}\left\{j \mid \mathbf{B}_{i, j}^{r}=1, j \in[N]\right\} .
$$

The desired view $\mathcal{U}$ requested by the user is also uniformly divided into $M_{u}$ blocks $\left\{\mathcal{U}_{i}, i \in\left[M_{u}\right]\right\}$. The coverage matrix of $\mathcal{U}$ is similarly defined as (1) by $\mathbf{B}^{u} \in \mathbb{R}^{M_{u} \times N}$, where $\mathbf{B}_{i, j}^{u} \stackrel{\text { def }}{=}$ $\mathbf{I}\left(\mathcal{U}_{i} \subseteq \mathcal{V}_{j}\right)$, and the set of cameras that cover $\mathcal{U}_{i}$ is denoted by $\lambda^{u}(i) \stackrel{\text { def }}{=}\left\{j \mid \mathbf{B}_{i, j}^{u}=1, j \in[N]\right\}$. We assume the coverage matrices represented by $\mathbf{B}^{r}, \mathbf{B}^{u}$ are known. Section 4 describes a practical approach to determine $\mathbf{B}^{r}, \mathbf{B}^{u}$ in the VSN we consider. The network provides user the desired view in a block-by-block manner. For each block $\mathcal{U}_{i}, i \in\left[M_{u}\right]$ in the desired view $\mathcal{U}$, the network selects a camera $\mathcal{C}_{s}$ satisfying the coverage requirement that $\mathbf{B}_{i, s}^{u}=1$ to transmit relevant data to the $\mathrm{CP}$ where an synthesized view of $\mathcal{U}_{i}$ is generated. We assume the energy required to transmit each block $\mathcal{R}_{i}$ is equal for all $i \in\left[M_{r}\right]$. The methods to extract relevant data and synthesize the desired view are described in Section 4.2.

We assume each block on the monitored plane is requested by the user independently throughout the operation of the network and the probability that block $\mathcal{R}_{i}$ is requested is given by $p_{i}$, where $\sum_{i=1}^{M_{r}} p_{i}=1$. We assume the CP uses a fixed selection rule to select cameras in response to user requests ${ }^{1}$, therefore, the lifetime $L$ of the network is a r.v. with a probability mass function (p.m.f) determined by $\left\{\mathbf{p}, \mathbf{w}, \mathbf{B}^{r}\right\}$, where $\mathbf{p}=\left[\begin{array}{llll}p_{1} & p_{2} & \ldots & p_{M_{r}}\end{array}\right]^{T}$ represent the distribution of users' requests and $\mathbf{w}=\left[\begin{array}{lllll}w_{1} & w_{2} & \ldots & w_{N}\end{array}\right]^{T}$ stands for the energy distribution in the network. The optimal energy allocation strategy is indicated by the energy distribution $\mathbf{w}^{*}$ that maximizes the expected value of network lifetime denoted by $\mathrm{E}\left[L\left(\mathbf{p}, \mathbf{w}, \mathbf{B}^{r}\right)\right]$, where $\sum_{i=1}^{N} \mathbf{w}_{i}^{*}=w_{t o t}, w_{t o t}$ denotes the total available energy, and $\mathrm{E}[\cdot]$ denotes the expectation operator.

In order to obtain a tractable formulation of our problem, we first map the energies of the cameras onto the monitored region and define the coverage energy of a block $\mathcal{R}_{i}$ as the sum of the energies of all the cameras that cover $\mathcal{R}_{i}$. Consider

$$
\mathbf{m}=\mathbf{B}^{r} \mathbf{w},
$$

where $\mathbf{m} \in \mathbb{R}^{M_{r}}$ and the $i^{t h}$ entry $m_{i}$ represents the coverage energy of $\mathcal{R}_{i}$. We next approximate the network lifetime as a function

\footnotetext{
${ }^{1}$ The optimal camera scheduling is considered in [7] using a closely related and compatible formulation.
}
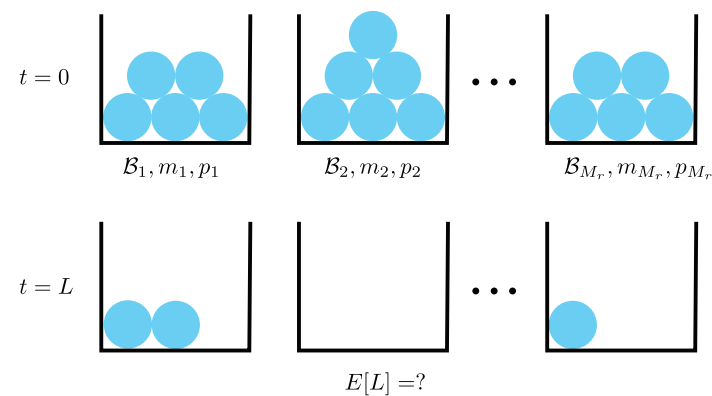

Fig. 2. An abstracted representation of the network lifetime. $M_{r}$ boxes $\left\{\mathcal{B}_{i}\right\}_{i=1}^{M_{r}}$ contains $m_{i}$ balls respectively. At each request, a ball is taken from $\mathcal{B}_{i}$ with probability $p_{i}$. After $L$ requests, one of these boxes first become empty. $\mathrm{E}[L]$ corresponds to the expected network lifetime.

of $\{\mathbf{m}, \mathbf{p}\}$ by using an abstraction for our problem as illustrated in Fig. 2. In this abstraction, we represent the $i^{\text {th }}$ monitored block $\mathcal{R}_{i}$ by a box $\mathcal{B}_{i}$ and the coverage energy of this block by $m_{i}$ balls. Each request of the block $\mathcal{R}_{i}$ on the monitored plane consumes energy equivalent to one ball. Since block $\mathcal{R}_{i}$ is utilized with probability $p_{i}$, the expected number of utilization after which the energy is entirely consumed can be represented by $\frac{m_{i}}{p_{i}}$ and correspondingly the expected lifetime can be given by $^{2}$

$$
\mathrm{E}[L(\mathbf{m}, \mathbf{p})] \approx \min \left(\frac{m_{1}}{p_{1}}, \frac{m_{2}}{p_{2}}, \ldots, \frac{m_{M_{r}}}{p_{M_{r}}}\right),
$$

This approximation in (4) is close provided the energy in each camera is large as compared to the energy for a single access and provided the difference between the two smallest values in $\left\{\frac{m_{i}}{p_{i}}\right\}_{i=1}^{M_{r}}$ is not negligible [7]. The optimal energy allocation is now defined as the strategy that maximizes the expected lifetime $\mathrm{E}[L(\mathbf{m}, \mathbf{p})]$.

Using (4), the optimal energy allocation $\mathbf{w}^{*}$ is obtained as the solution to the following max-min optimization problem,

$$
\begin{gathered}
\max _{\mathbf{w}} \min _{i}\left\{f_{i}\right\}_{i=1}^{M_{r}} \\
\text { s.t. } \sum_{i=1}^{N} w_{i}=w_{\text {tot }} \\
\mathbf{f}=\mathbf{P B}^{r} \mathbf{w} \\
w_{i} \geq 0
\end{gathered}
$$

where $f_{i} \stackrel{\text { def }}{=} \frac{m_{i}}{p_{i}}$ for $i=1,2, \ldots, M_{r}$ and $\mathbf{f}=\left[f_{1}, f_{2}, \ldots, f_{M_{r}}\right], \mathbf{P}$ is the diagonal matrix formed by the vector $\left[\frac{1}{p_{1}}, \frac{1}{p_{2}}, \ldots, \frac{1}{p_{M_{r}}}\right]$.

\section{REFORMULATION OF OPTIMAL ALLOCATION AS A LINEAR PROGRAMMING PROBLEM}

It can be seen that our formulation above corresponds to maximization of a concave function. Although numerical routines are available to address the optimization problem (5), it is not well-behaved partly due to the fact that the objective function in (5) is not differentiable everywhere, motivating our search for more efficient algorithms to solve (5)

We next transform the optimization in (5) into a LP problem, for which efficient algorithms can achieve accurate solution in polyno-

\footnotetext{
${ }^{2} \mathrm{~A}$ more formal analysis leading to the approximation is presented in [7]
} 


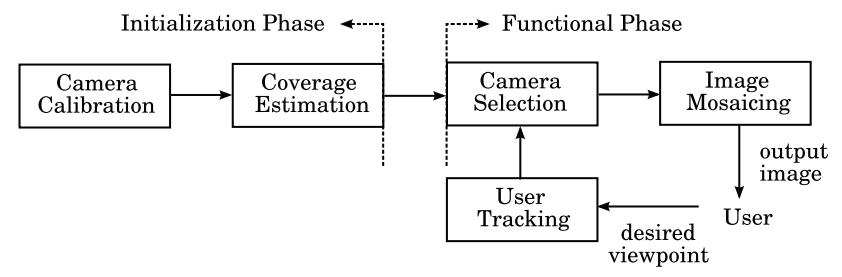

Fig. 3. The operation of the network.

mial time [8]. Introducing a new variable $t$, we rewrite the max-min optimization problem of (5) as the equivalent linear programming problem:

$$
\begin{aligned}
& \min _{\mathbf{w}} t \\
& \text { s.t. } \sum_{i=1}^{N} w_{i}=w_{t o t} \\
& \quad \mathbf{f}=\mathbf{P B}^{r} \mathbf{w} \\
& \quad-f_{i} \leq t, w_{i} \geq 0, \text { for } i=1,2, \ldots, M_{r}
\end{aligned}
$$

The LP (6) can be rewritten in standard LP form by defining $\mathbf{u} \stackrel{\text { def }}{=}\left[\mathbf{w}^{T}, t\right]^{T}$, thus the objective function can be written as $\mathbf{h}^{T} \mathbf{u}$ where $\mathbf{h} \stackrel{\text { def }}{=}\left[\mathbf{0}^{T}, 1\right]^{T}$ and $\mathbf{0}$ denotes a $N \times 1$ vector with value 0 . The constraints in (6) can also be rewritten in terms of $\mathbf{u}$. The optimal values of $\mathbf{w}^{*}, t^{*}$ are obtained simultaneously by solving the LP problem for $\mathbf{u}^{*}=\left[\mathbf{w}^{* T}, t^{*}\right]$.

\section{SYSTEM SCENARIO}

We describe the implementation details of the VSN illustrated in Fig. 1. In the initialization stage, the coverage information $\mathbf{B}^{u}$ and $\mathbf{B}^{r}$ for the image sensors is obtained. In the functional stage, for each of desired view $\mathcal{U}$ requested by users, the network selects a subset of the cameras to provide the visual data. The synthesized view $\tilde{\mathcal{U}}$ is generated at the $\mathrm{CP}$ using received data.

\subsection{Initialization Stage}

Coverage information can be obtained by estimating parameters of the cameras deployed in the network, commonly referred to as camera calibration [9]. Using a homogeneous representation, the image coordinate $\mathbf{x}=\left[\begin{array}{lll}x_{1} & x_{2} & x_{3}\end{array}\right]^{T}$ of a $3 D$ point $\mathbf{X}=\left[\begin{array}{llll}X_{1} & X_{2} & X_{3} & 1\end{array}\right]^{T}$ is given by $\mathbf{x}=\mathcal{P} \mathbf{X}$, where $\mathcal{P} \in \mathbb{R}^{3 \times 4}$ denotes the camera projection matrix [9] which is determined by intrinsic parameters (such as focal length) and extrinsic parameters (orientation and location) of the camera. We use plane-based camera calibration methods $[10,11]$ to estimate these camera parameters.

The coverage matrices $\mathbf{B}^{r}$ and $\mathbf{B}^{u}$ are estimated after calibrating the cameras. A world point $\mathbf{X}$ is covered by a camera $\mathcal{C}_{s}$ if the image coordinate of this point $\mathrm{x}$ lies within the FoV of this camera $\mathcal{V}_{s}$. An example of coverage estimation can be found in Fig. 1. Now given the total available energy $w_{t o t}$, the distribution of user requests $\mathbf{P}$, and the coverage matrix $\mathbf{B}^{r}$, an energy allocation procedure is invoked to distribute $w_{\text {tot }}$ among $N$ cameras and obtain $\mathbf{w}$. We have $\mathbf{w}=\mathbf{w}^{*}$ for our optimal allocation procedure.

\subsection{Functional Stage}

For each block $\mathcal{U}_{i}$ in the desired view $\mathcal{U}$, we select a camera $\mathcal{C}_{s}$ in order to capture and transmit relevant data. This camera is selected according to a camera selection strategy described in [7], which attempts to maximize the remaining network lifetime by maximizing $\mathrm{E}[L(\mathbf{m}, \mathbf{p})]$.

At the $\mathrm{CP}$, the raw data for each desired block $\mathcal{U}_{i}$ must be transformed and mosaiced together to synthesize the desired view. The mosaicing is described by a homography [9] for the texture image of a planar surface (our case). Without loss of generality, we assume $X_{3}=0$ for all world points $\mathbf{X}$ on the monitored plane and let $\mathbf{X}_{h}=\left[\begin{array}{lll}X_{1} & X_{2} & 1\end{array}\right]^{T}$ where we neglect $X_{3}=0$, represents a world point on this plane. The image coordinate $\mathrm{x}$ can be written as: $\mathbf{x} \sim \mathcal{H} \mathbf{X}_{h}$, where $\sim$ indicates equality up to a scale factor and $\mathcal{H} \in \mathbb{R}^{3 \times 3}$ is a matrix denoting the homography between the camera plane and the target plane. $\mathcal{H}$ can be calculated from the parameters of calibrated cameras. Let $\mathbf{x}_{1}, \mathbf{x}_{2}$ denote the image coordinates of world point $\mathbf{X}_{h}$ in two cameras, then the following equality holds $\mathcal{H}_{1}^{-1} \mathbf{x}_{1}=\mathbf{X}_{h}=\mathcal{H}_{2}^{-1} \mathbf{x}_{2}$, thus $\mathbf{x}_{2}$ can be obtained from $\mathbf{x}_{1}$ as $\mathbf{x}_{1}=\mathcal{H}_{1} \mathcal{H}_{2}^{-1} \mathbf{x}_{2}$. This relation allows the view at user's desired viewpoint to be rendered from corresponding regions in the selected camera. The $\mathrm{CP}$ also keeps a record of previous user requests in order to obtain an estimate of the distribution of these requests, if this distribution is not provided as a priori.

\section{SIMULATION}

We perform simulations to validate the proposed energy allocation scheme. $N$ cameras are placed randomly within a $4 m \times 3 m$ field located $3 m$ from the target plane $\mathcal{R}$ which is of size $4 m \times 3 m$ (typical size of a wall). Users' viewpoints are generated according to a separable Gaussian distribution with its maxima at the center of the wall, with a standard deviation of $0.3 \mathrm{~m}$ along the two dimensions. The probabilistic distribution of each block on the monitored plane being requested, i.e. $\mathbf{p}$, is obtained by a Monte-Carlo simulation. All cameras and users' views point toward the target plane with a random rotation up to \pm 0.1 radian along each of the three axes. We further assume all cameras and the users' viewpoints have images of $200 \times 200$ (in pixel units). The camera images are rendered by using the scene geometry (homography).

We first conduct a Monte Carlo simulation in order to determine the number of cameras required for adequate coverage of the target plane [6]. We found that using a focal length $f_{0}=427.5$ (in pixel units) ${ }^{3}$, a minimum of 50 cameras are necessary in order to ensure (with $99 \%$ confidence) that the target plane is covered in the beginning. We thus use $N=100$ cameras to provide enough coverage redundancy.

We simulate a scenario that the total available energy $w_{t o t}$ is $50 \mathrm{~J}$, and the transmission of a frame of camera image costs $1 J$ of energy. At each time instant, a desired view is requested. We generate 100 user's viewpoints in each simulation and present averages over 100 simulations. Two energy allocation schemes are compared,

- Optimized allocation by solving (6) using LP (LinOpt).

- Uniform distribution of the total available energy $w_{t o t}$ among all the cameras (UniForm).

Figure 4 compares the percentage coverage on the target plane over time for the energy allocation schemes above. The figure clearly

${ }^{3}$ For an image sensor with size $35 \mathrm{~mm} \times 35 \mathrm{~mm}$, this would correspond to focal length of $75 \mathrm{~mm}$. 


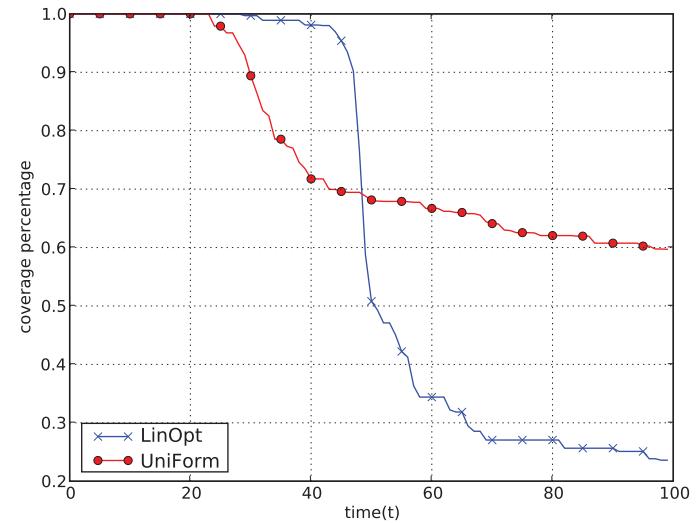

Fig. 4. Comparison of coverage using different energy allocation schemes.

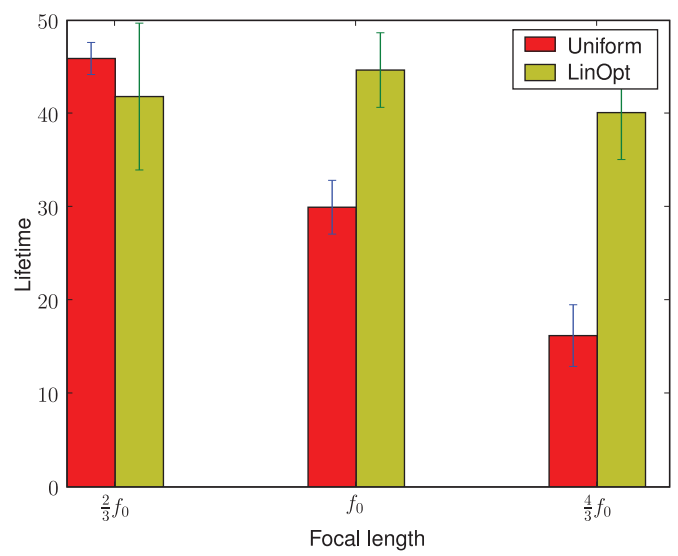

Fig. 5. Comparison of lifetime using different focal lengths.

illustrates the benefit of the proposed optimized energy allocation. When the lifetime is defined as the duration over which $95 \%$ of the monitored area is covered, LinOpt prolongs the network lifetime by a factor of almost 2 compared to the unoptimized UniForm scheme. Direct solution of the max-min problem (5) yields performance very close to LinOpt (as expected) but requires much more computation (2.3s v.s. $157 \mathrm{~s}$ for Matlab ${ }^{\mathrm{TM}}$ based implementation).

For the different energy allocation schemes, Fig. 5 examines the effect of the focal length of the cameras on the network lifetime for focal lengths $\frac{2}{3} f_{0}, f_{0}$, and $\frac{4}{3} f_{0}$. We observe from Fig. 5 that energy allocation using LinOpt is more effective as the focal length increases. When the focal length is small, LinOpt and UniForm have similar performance. This behavior can be anticipated because as the focal length decreases, the fields of view of the cameras become enlarged resulting in greater overlap and consequently less benefit from the optimal allocation. In the extreme case when each camera covers the entire target area, the lifetime is independent of energy allocation.

\section{DISCUSSION AND CONCLUSION}

In this work, we propose a probabilistic formulation for the lifetime of a user-centric image sensor network, based on which the lifetime-maximizing energy allocation strategy is formulated as a linear programming problem. The proposed formulation explicitly address the unique characteristics of the coverage problem in VSNs, and stochastic user interactions. Simulation results demonstrate the advantage of the proposed methodology over a naive uniform allocation scheme.

We conclude this paper with a few remarks. Firstly, although we consider a simple application, the analysis can be extended to other VSN applications, e.g. a surveillance system designed to capture moving objects. In this scenario, the coverage information $\mathbf{B}^{r}, \mathbf{B}^{u}$ can be estimated by suitably discretizing the monitored area (e.g. $3 D$ space can be divided into small cubes). The rest of the analysis is identical with that presented in this paper. Secondly, our formulation maps the energy from the cameras onto the target area in (3). Note that in this process, we are neglecting the fact that, at each user request, the change in the energy of the selected camera $\mathcal{C}_{s}$, will in fact change the energy distribution not only over the block being requested, which we shall account for, but also over the other blocks for which $\mathcal{C}_{s}$ provides coverage. As a result, our approach yields sub-optimality. However, this sub-optimality is mitigated when the focal lengths of the sensors are reasonably large as demonstrated by our simulations.

\section{REFERENCES}

[1] A. Keshavarz, A. Tabar, and H. Aghajan, "Distributed vision-based reasoning for smart home care," in Proceedings of ACM SenSys Workshop on Distributed Smart Cameras (DSC06), 2006.

[2] M. Wu and C. Chen, "Collaborative Image Coding and Transmission over Wireless Sensor Networks," EURASIP Journal on Advances in Signal Processing, special issue on visual sensor networks, 2007.

[3] Y. Chen and Q. Zhao, "On the lifetime of wireless sensor networks," IEEE Commun. Lett., vol. 9, no. 11, pp. 976-978, 2005.

[4] C. Huang and Y. Tseng, "The Coverage Problem in a Wireless Sensor Network," Mobile Networks and Applications, vol. 10, no. 4, pp. 519-528, 2005.

[5] J. Dagher, M. Marcellin, and M. Neifeld, "A method for coordinating the distributed transmission of imagery," IEEE Trans. Image Proc., vol. 15, no. 7, pp. 1705-1717, Jul. 2006.

[6] C. Yu, S. Soro, G. Sharma, and W. Heinzelman, "Lifetime-distortion trade-off in image sensor networks," in Proc. IEEE Intl. Conf. Image Proc., vol. V, Sept. 2007, pp. 129-132.

[7] C. Yu and G. Sharma, "Sensor scheduling for lifetime maximization in usercentric image sensor networks," in Proc. SPIE: Visual Communications and Image Processing (VCIP), M. Rabbani and R. L. Stevenson, Eds., vol. 7257, Jan. 2009 pp. $7257-0 \mathrm{H}-1-12$.

[8] D. G. Luenberger, Linear and Nonlinear Programming, 2nd ed. Reading, MA: Addison Wesley, 1989.

[9] R. Hartley and A. Zisserman, Multiple view geometry in computer vision. New York, NY, USA: Cambridge University Press, 2000.

[10] Z. Zhang, "Flexible camera calibration by viewing a plane from unknown orientations," in IEEE Intl. Conf. Comp. Vision., 1999, pp. 666-673.

[11] C. Yu and G. Sharma, "Plane-based calibration of cameras with zoom variation," in Proc. SPIE: Visual Communications and Image Processing, J. G. Apostolopoulos and A. Said, Eds., vol. 6077, 15 - 19 Jan. 2006, pp. 607 710-1-607 710-9. 